INRA Prod. Anim.,

2014, 27 (5), 331-336

\title{
Sélection génomique : quelles perspectives pour les filières avicoles?
}

\author{
P. LE ROY ${ }^{1,2}$, H. CHAPUIS ${ }^{3}$, D. GUÉMENÉ \\ 1 INRA, UMR1348 PEGASE, F-35590 Saint-Gilles, France \\ 2 Agrocampus Ouest, UMR1348 PEGASE, F-35042 Rennes, France \\ 3 SYSAAF, UR83 Recherches Avicoles F-37380 Nouzilly, France
}

Courriel : pascale.leroy@rennes.inra.fr

En quelques années, la sélection génomique a été mise en place chez les bovins laitiers. Réformant méthodes et organisations, elle a démontré toute sa capacité à apporter des gains génétiques importants. Les opérateurs de la sélection dans les filières avicoles ont assisté à cette « révolution » et se posent légitimement la question de l'intérêt pour eux d'entrer dans cette démarche.

L'association préférentielle, sur un même segment chromosomique, de certains allèles de marqueurs moléculaires avec l'allèle favorable à un gène influençant un caractère, permet d'envisager une forme de sélection qui se substituerait à la « boîte noire » proposée par Fischer (1918). Dès 1990, Lande et Thompson (1990) proposent d'exploiter ce déséquilibre d'association gamétique, encore appelé Déséquilibre de Liaison (DL) entre marqueurs et «Quantitative Trait Loci » (QTL), pour estimer la valeur génétique des animaux en utilisant un " score moléculaire ». Sur ce principe de Sélection Assistée par Marqueurs (SAM), différentes méthodes d'évaluation génétique ont été développées (Fernando et Grossmann 1989, Goddard 1992). Cependant, la densité des marqueurs disponibles à cette époque est insuffisante pour que le DL puisse être exploité efficacement (Ruane et Colleau 1995) : les associations sont valides au sein des familles mais pas au sein de la population entière ; les associations sont valides pour un nombre limité de générations à cause des recombinaisons entre marqueurs et QTL ; les QTL associés aux marqueurs n'expliquent pas toute la variabilité génétique observée. Pour résoudre tous ces défauts, Haley et Visscher (1998) proposent d'utiliser plusieurs milliers de marqueurs moléculaires couvrant l'ensemble du génome. C'est le début de la "sélection génomique ». Le séquençage des génomes des espèces d'animaux domestiques (Vignal 2011) va rendre l'idée réalisable au cours des années 2000 grâce à la mise en évidence de centaines de milliers de marqueurs SNP ("Single Nucléotide Polymorphisms »). Si le premier génome rendu public est celui de la poule, en 2004, c'est chez le bovin, séquencé deux ans plus tard, que l'utilisation d'outils génomiques va révolutionner les schémas de sélection. A partir de 2007, la disponibilité des puces bovines $54 \mathrm{~K}$ (sur lesquelles 54000 marqueurs SNP sont génotypés) va permettre la mise en œuvre d'une sélection génomique en grandeur réelle dans les grandes races laitières (Guillaume et al 2011). Les résultats obtenus chez les bovins ont pleinement validé les gains potentiels apportés par cette nouvelle stratégie de sélection. Ils ont aussi fourni certaines règles à suivre pour faire une prédiction génomique précise (RobertGranié et al 2011).

\section{1 / Les principes de la sélection génomique}

\section{1 / Une sélection en deux étapes}

En principe, la sélection génomique suppose deux étapes distinctes. Les effets des génotypes aux marqueurs SNP sur les phénotypes sont tout d'abord estimés sur un ensemble d'animaux génotypés et phénotypés. Ceux-ci constituent la « population de référence ». Une fois l'équation de prédiction établie sur la base de ces effets estimés, il est possible, et c'est la seconde étape, de l'appliquer à la population des candidats à la sélection. Ceux-ci peuvent ne pas être phénotypés et leur pedigree peut ne pas être connu, leur valeur génomique dépendant uniquement de leur génotype aux marqueurs SNP. Elle se calcule simplement en sommant les effets associés aux génotypes aux marqueurs SNP dont les candidats sont porteurs. Il est ainsi possible de prédire la valeur génétique d'un individu dès sa naissance (en pratique dès que son ADN peut être extrait), avant de connaître ses performances ou celles de ses descendants, avec un niveau de précision relativement élevé et, en tous cas, très supérieur à celui d'un index sur ascendance.

En pratique, le GBLUP ( Genomic Best Linear Unbiased Predictor »), ou BLUP génomique, suppose que tous les SNP affectent potentiellement le phénotype et estime $p$ effets de SNP. Il consiste à remplacer $\mathbf{A}$, la matrice de parenté généalogique déduite des pedigrees par $\mathbf{G}$, une matrice de parenté génomique (Van Raden 2008, Goddard 2009). G serait considérée comme la matrice des parentés « réalisées », tandis que A serait celle des parentés « attendues ». Comme l'ont montré Habier et al (2007), quelle que soit la méthode utilisée pour estimer les effets des SNP, celle-ci exploite à la fois le gain de précision sur les liens de parenté entre les individus apporté par les marqueurs et l'information sur le DL entre marqueurs et QTL. Si 1000 à 2000 marqueurs sont suffisants pour préciser les liens de parenté, une densité en marqueurs plus importante permet en plus de capturer le DL. Partant d'une population de référence, au cours des générations de sélection, le gain de précision sur la parenté apporté par les marqueurs va rapidement décroître, alors que l'effet du DL va se maintenir plus longtemps, d'autant plus que la densité sera grande, conférant une supériorité à la sélection génomique par rapport à la sélection sur indice BLUP traditionnelle. Enfin, une approche « single step » a également été proposée par Aguilar et al (2010). Elle permet d'envisager une analyse où sont combinées de façon optimale les informations provenant de tous les individus, 
qu'ils soient ou non phénotypés et/ou génotypés.

\section{2 / Estimer l'effet des SNP}

L'estimation d'un très grand nombre d'effets de SNP ( $p$ variables) à partir d'une population de référence de taille limitée ( $n$ observations) est délicate, ce que les statisticiens appellent un problème de grande dimension $\langle p>>n »$. Différents modèles, qui formulent des hypothèses distinctes sur la distribution des effets des SNP, ont été développés pour résoudre cette difficulté. A la différence du GBLUP qui estime $p$ effets de SNP, les algorithmes d'apprentissage automatique (Long et al 2007) vont rechercher quels sont les quelques SNP qui affectent fortement le phénotype et utiliser uniquement ces derniers dans l'équation de prédiction. Les méthodes Bayésiennes sont à michemin, en supposant l'existence de quelques SNP avec des effets importants, d'un grand nombre avec un petit effet et d'autres, nombreux également, avec un effet explicitement nul. Le Bayes B proposé par Meuwissen et al (2001), et plus récemment le Bayes $\mathrm{C} \pi$ (Habier et al 2011), sont considérés comme des références en termes d'efficacité pour la prédiction génomique, malgré des coûts de calculs considérables.

\section{3 / Déséquilibre de liaison et population de référence}

Afin d'améliorer l'informativité des marqueurs, et donc d'accroître le DL entre marqueurs et QTL, il est possible d'étudier non plus les effets des SNP individuellement mais les effets de blocs de quelques SNP (Guillaume et al 2009). Cependant, connaissant les génotypes aux marqueurs SNP, cette stratégie nécessite d'établir quelles sont les combinaisons d'allèles, appelées haplotypes, portées par chacun des chromosomes homologues. Toutefois, Solberg et al (2008) soulignent qu'établir quels sont les haplotypes portés par les individus ajoute un terme d'erreur et peut nuire à la précision. Le niveau de DL peut aussi être accru en augmentant la densité des puces SNP utilisées. Cependant, ce gain théorique doit être estimé en considérant aussi les coûts de calculs, les méthodes Bayésiennes devenant difficilement applicables avec des puces à très haute densité. Par ailleurs, plusieurs études de simulation ont montré une asymptote dans le gain de précision lié à l'augmentation du nombre de marqueurs (Sonesson et Meuwissen 2009).

Concernant la population de référence, il est d'usage de considérer qu'au moins 1000 individus sont nécessaires pour que la relation entre génotype et phénotype soit suffisamment fiable. La population de référence doit notamment être suffi- samment large pour contenir un échantillon représentatif de la population, où les « bons » comme les « mauvais » sont représentés, sous peine de biaiser les équations de prédiction. Par ailleurs, il faut également une grande " proximité » génétique entre cette population de référence et la population à sélectionner (Legarra et al 2008, Habier et al 2011). Cela implique d'entretenir une population de référence régulièrement renouvelée, avec les coûts associés, sous peine de laisser le DL se rompre au fil des générations successives. En effet, l'hypothèse initialement avancée selon laquelle il suffirait de génotyper une fois une population de référence pour se passer par la suite des couteuses opérations de phénotypage (Soller 1994) s'est révélée irréaliste. En témoigne notamment la chute de la précision des valeurs génétiques dès qu'on cesse le phénotypage (Sonesson et Meuwissen 2009), chute ralentie toutefois par une densité accrue des marqueurs (Solberg et al 2009).

\section{2 / La validation des évalua- tions génomiques}

\section{1 / Etudes par simulation}

Après la présentation conceptuelle de Meuwissen et al (2001), et avant la publication des premiers résultats issus d'applications dans des schémas de sélection chez les bovins laitiers, de nombreux travaux basés sur des simulations ont été réalisés afin de juger de la pertinence de l'évaluation génomique. Le principe général consiste à simuler, pour chaque individu de la population étudiée, un génome de taille arbitraire (par exemple 10 chromosomes de $100 \mathrm{cM}$ ). Afin de créer un DL entre SNP et QTL, la population est renouvelée sans sélection pendant un grand nombre de générations (entre 1000 et 5000 générations selon les auteurs) en tenant compte des recombinaisons et des mutations. Une sélection génomique en deux étapes est ensuite simulée et l'efficacité de la sélection est évaluée en comparant les Valeurs Génomiques Estimées (VGE) et les Valeurs Génétiques Vraies (VG) qui sont ici connues. Cette analyse fournit un coefficient de régression $(b)$ et un coefficient de corrélation $(r)$ entre VGE et VG. Le coefficient de corrélation entre VGE et VG reflète la précision de l'évaluation génomique. Le coefficient de régression traduit le biais de l'estimation, $b=1$ signifiant que l'estimation est sans biais, $b>1$ que les VGE sont systématiquement surévaluées et $b<1$ qu'elles sont systématiquement sous-évaluées.

Les études par simulation ont permis de confirmer la supériorité de la sélection génomique sur la sélection classique sur pedigree. Elles ont également fourni des évolutions au cours des générations qui soulignent la nécessité de renouveler la population de référence, d'une part, et de procéder à des étapes de validation, d'autre part (Robert-Granié et al 2011). Il faut toutefois remarquer que les effets des SNP simulés au cours de ces études sont en général en accord avec les hypothèses faites lors du développement des modèles d'évaluation génomique décrits précédemment (cf. §1.2) (nombre de QTL affectant le caractère, distribution des effets, additivité des effets).

\section{2 / Etudes rétrospectives}

Les premières générations de sélection génomique ont également permis d'estimer la validité des évaluations génomiques à partir de données réelles. Des évaluations croisées ont été réalisées au travers d'études rétrospectives : en se replaçant quelques années en arrière, les valeurs génomiques estimées à l'époque pour de jeunes reproducteurs sont comparées à leurs valeurs génétiques estimées à partir des performances moyennes de leurs descendances connues aujourd'hui. De telles études rétrospectives ont été entreprises sur des données disponibles dans différentes espèces, souris (Legarra et al 2008), bovins laitiers (Fritz et al 2010, Colombani et al 2012) et poule (Wolc et al 2011a, Wolc et al 2011b).

Le premier article de Wolc et al (2011b) décrit les résultats d'une évaluation génomique mise en œuvre sur 16 caractères, dans une population de poules pondeuses. La population de référence était constituée de 2708 animaux, dont 1563 poules, génotypées pour $23356 \mathrm{SNP}$ informatifs. Sauf pour l'âge de la maturité sexuelle, le gain de précision des index génomiques est manifeste, la précision des VGE étant jusqu'à doublée dans les cas les plus favorables. Il apparaît clairement que le classement des individus et les décisions de sélection seraient modifiés par l'utilisation d'index génomiques par rapport à des BLUP classiques. En revanche, sur ces données réelles, le choix de la méthode de calcul (GBLUP vs Bayes $\mathrm{C} \pi$ ) des index génomiques n'a que peu d'impact, ce résultat étant cohérent avec les observations similaires faites chez les bovins laitiers (Colombani et al 2012). Les index génomiques étant calculés dans des analyses uni-caractères, ils peuvent être légèrement biaisés, mais ce biais est de moindre importance avec la méthode Bayes $\mathrm{C} \pi$.

Dans le second article, Wolc et al (2011a) ont étudié comment évoluait la précision des valeurs génomiques sur une période de 5 générations successives en se basant, soit sur une information généalogique, soit sur les marqueurs. Comme on pouvait s'y attendre, la précision (que 
l'on s'attend à voir décroître d'un facteur $\sqrt{1 / 2}$ à chaque génération) est quasi nulle pour de nombreux caractères si on ignore l'information génomique, alors qu'elle demeure significative en utilisant la méthode Bayes $\mathrm{C} \pi$. Les caractères les plus robustes sont ceux qui ont l'héritabilité la plus élevée ou ceux qui sont influencés par un faible nombre de QTL.

\section{3 / L'efficacité attendue de la sélection génomique}

\section{1 / Les sources du progrès géné- tique}

Le progrès génétique annuel $\Delta \mathrm{G}$ est défini comme le gain de valeur génétique observé en moyenne dans la population en un an. Son expression dépend de quatre paramètres : la variabilité génétique du caractère à améliorer, l'intensité de sélection appliquée, la précision des valeurs génétiques estimées et l'intervalle de génération. La sélection génomique peut permettre des gains de progrès génétique en jouant sur les trois derniers paramètres, la variabilité génétique étant une donnée biologique.

Certains caractères, tels la résistance aux maladies ou les qualités sensorielles des produits, peuvent se révéler couteux à mesurer à grande échelle. Le nombre de candidats à la sélection pour lesquels une information phénotypique est disponible peut alors être faible. L'intensité de sélection pourra être augmentée par l'évaluation génomique, en élargissant la base de sélection, si le génotypage est plus facile à mettre en œuvre que le phénotypage.

L'évaluation génomique peut également permettre d'estimer plus précisément la valeur génétique d'un candidat. D'après Hayes et al (2009), la précision de l'évaluation génomique repose sur quatre facteurs : le niveau de DL entre les marqueurs et les QTL, la taille de la population de référence, l'héritabilité des caractères sélectionnés et leur architecture génétique (y a-t-il quelques gènes à effet forts ou de nombreux gènes à petits effets ?). Nous avons vu précédemment (cf. § 1.3), comment DL et taille de la population de référence agissent sur la précision des effets SNP estimés et, par conséquent, sur la précision des valeurs génomiques calculées. La figure 1 montre comment évolue la précision des valeurs génomiques avec la taille de la population de référence et en fonction de l'héritabilité (Tribout et al 2011). Ces courbes illustrent le manque de précision de l'évaluation génomique pour les caractères faiblement héritables. Cependant, chaque fois que le phénotype informe peu sur la valeur génétique, d'importants gains de

Figure 1. Précision (CD) des valeurs génomiques estimées en fonction de la taille de la population de référence et de l'héritabilité $\left(h^{2}\right)$ du caractère considéré, pour une population de taille efficace $(\mathrm{Ne})=100$. D'après Tribout et al (2011).

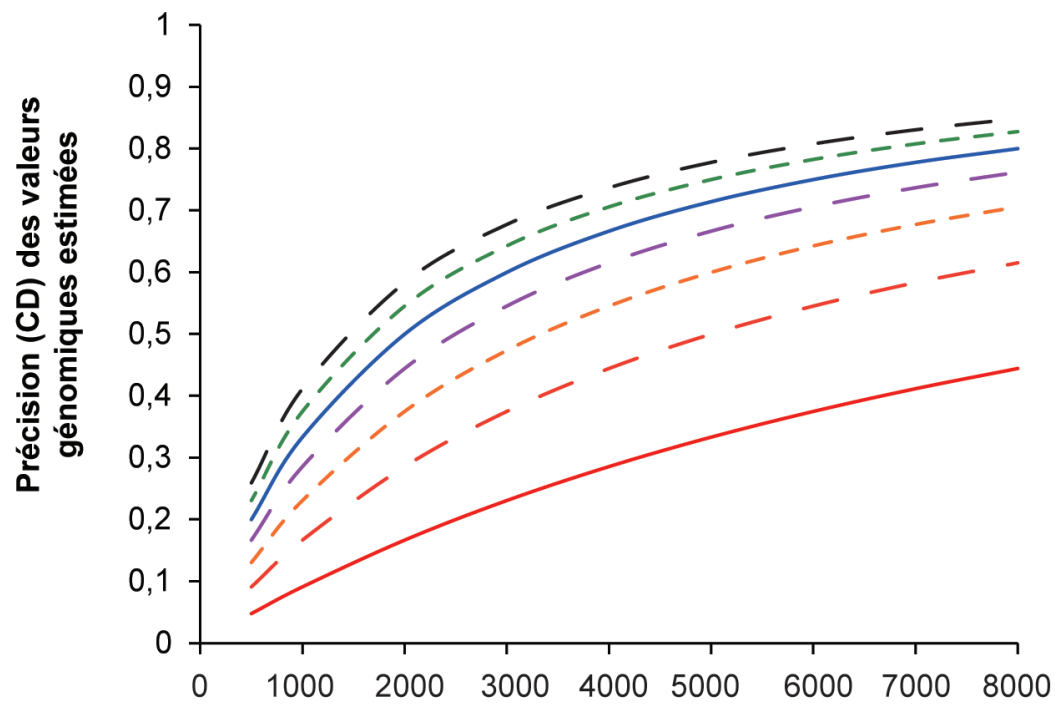

Taille de la population de référence

$$
\begin{gathered}
-\mathrm{h}^{2}=0,7---\mathrm{h}^{2}=0,6-\mathrm{h}^{2}=0,5--\mathrm{h}^{2}=0,4 \\
----\mathrm{h}^{2}=0,3--\mathrm{h}^{2}=0,2-\mathrm{h}^{2}=0,1
\end{gathered}
$$

Figure 2. Gain de progrès génétique apporté par la SAM en fonction de la part de variance génétique expliquée par les QTL et de l'héritabilité $\left(h^{2}\right)$ du caractère. L'axe des ordonnées est en unités de progrès génétique obtenu par sélection sur pedigree. D'après Lande et Thompson (1990).

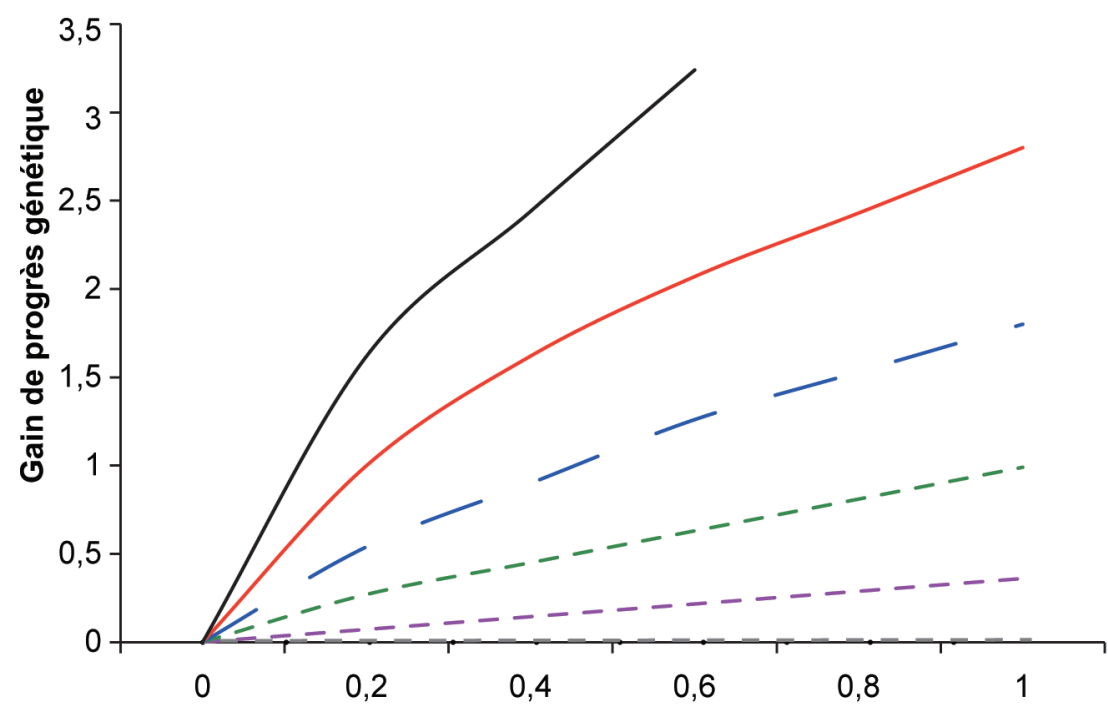

Part de variance génétique expliquée par les QTL

$$
\begin{aligned}
-h^{2} & =0,025-h^{2}=0,05-h^{2}=0,10 \\
---h^{2} & =0,20---h^{2}=0,5---h^{2}=1,0
\end{aligned}
$$

précision peuvent être obtenus par une évaluation génomique en comparaison d'une évaluation sur pedigree (Lande et Thompson 1990). Ce sera notamment le cas des caractères faiblement héritables (fertilité) ou nécessitant un contrôle sur descendance (ponte) ou collatéraux (qua- lité de la viande). La figure 2 montre le gain de progrès génétique attendu en fonction de l'héritabilité et de la part de variance génétique expliquée par les marqueurs. L'utilisation de puces SNP à très haute densité couvrant l'ensemble du génome, permet aujourd'hui d'espérer 
expliquer toute la variance génétique dans l'équation de prédiction, c'est-à-dire de se situer au maximum de la variance expliquée sur la figure 2 . Le progrès génétique pourrait donc être doublé pour un caractère d'héritabilité égale à 0,20 et pratiquement multiplié par trois pour un caractère d'héritabilité 0,10 . A l'inverse, aucun gain substantiel n'est à attendre pour les caractères d'héritabilité forte (composition corporelle).

Enfin, la sélection génomique permet d'envisager une réduction de l'intervalle de génération en obtenant une précision raisonnable pour les indices avant l'étape de phénotypage. Si l'intérêt est évident dans le contexte des bovins laitiers, il peut également être réel dans les schémas de sélection avicole pour les poules pondeuses en permettant une sélection précoce des coqs, ou la prise en compte de nouveaux caractères peu héritables ou difficilement mesurables (caractères de santé et de bien-être animal). En revanche, le gain attendu ne semble pas être à la hauteur des coûts pour des caractères de croissance (poids vif) mesurés précocement et généralement héritables, donc efficacement sélectionnés par les méthodes classiques.

Par ailleurs, dans les populations fermées d'effectif limité où le maintien d'une variabilité génétique suffisante demeure une question importante, la sélection génomique permet, en caractérisant mieux l'aléa de méiose, de ne pas surreprésenter des pleins-frères/sœurs et d'aller mieux échantillonner les candidats retenus dans différentes familles, ce qui contribue à ralentir l'augmentation de la consanguinité (Daetwyler et al 2007) et donc à maintenir la variabilité génétique.

\section{2 / Les particularités des volailles}

Une caractéristique des schémas de sélection avicole réside dans l'utilisation du croisement (figure 3). Or, il est pratiquement délicat et couteux de sélectionner efficacement les noyaux de sélection (« lignées pures ») pour les performances en croisement (« poules commerciales ») car cela nécessite un suivi du pedigree. Une sélection assistée par marqueurs utilisant l'information des croisements pour sélectionner dans les noyaux de sélection a été proposée (Dekkers et Chakraborty 2004). L'utilisation de la sélection génomique permet en théorie une meilleure prise en compte des phénomènes non additifs (hétérosis, dominance) attendus en croisement (Dekkers 2007). Elle soulève néanmoins des questions supplémentaires si les effets des SNP sont différents d'une population à l'autre (Kinghorn et al 2010). IbanezEscriche et al (2009) ont toutefois montré qu'avec une densité de marqueurs

Figure 3. Exemple d'un schéma de multiplication de lignées de poules pondeuses.

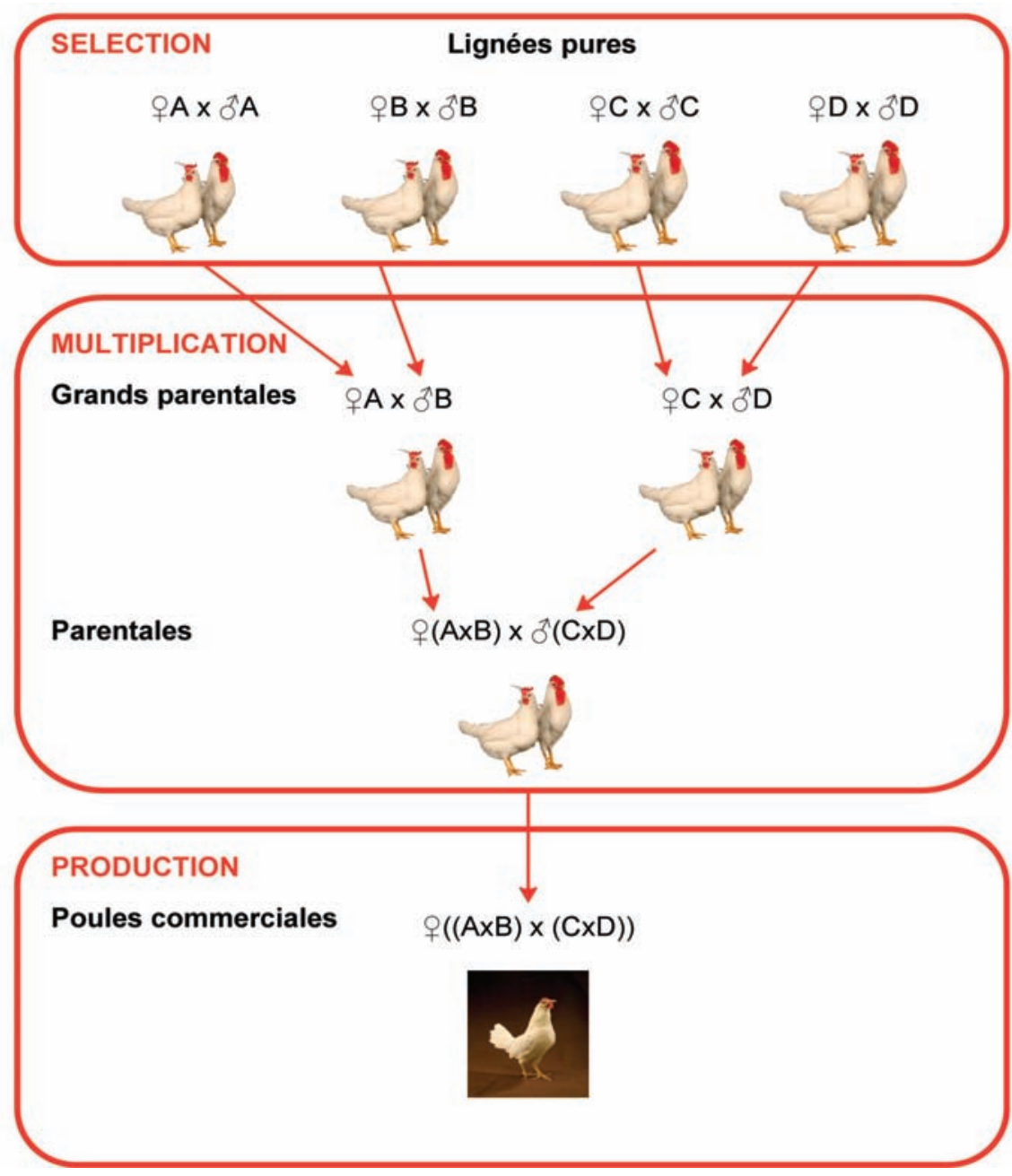

suffisante, il n'était pas nécessaire de développer un modèle " populationspécifique » et que cela ouvrait d'intéressantes perspectives pour l'amélioration des performances des descendants croisés sans nécessiter un suivi du pedigree.

La structure pyramidale des schémas de sélection des volailles implique également des différences d'environnements entre étages de sélection et de production. Au-delà, la sélection d'animaux pour l'exportation pose le problème de la stabilité des performances lorsque le milieu varie. Ce problème classique des interactions génotype $\times$ milieu peut lui aussi être revisité à la lumière de l'évaluation génomique. En déconnectant le calcul de la valeur génétique de l'obtention d'un phénotype, l'évaluation génomique est en effet une stratégie permettant de sélectionner dans un environnement en ciblant la production dans un autre.

\section{Conclusion}

En quelques années, la sélection génomique a été mise en place chez les bovins laitiers, tant au niveau national que dans le cadre de l'évaluation inter- nationale des reproducteurs. Les opérateurs de la sélection dans les autres filières animales ont assisté à cette révolution et se posent légitimement la question de l'intérêt pour eux d'entrer dans cette démarche (Coudurier 2011).

La disponibilité d'une puce « poule » de 600k SNP permet d'ores et déjà d'appliquer une sélection génomique dans les filières poule pondeuse et poulet de chair. Cependant, contrairement aux bovins laitiers, la sélection des lignées avicoles est réalisée dans des populations de taille modeste. Bien que peu d'études soient disponibles pour juger de l'étendue du DL dans ces populations, la densité disponible avec 600k SNP devrait permettre une évaluation multiraciale efficace (De Roos et al 2009). Au-delà, le partage des informations entre opérateurs concurrents présenterait un réel intérêt, comme démontré dans le cas de la race bovine Holstein (Lund et al 2010). Pour les autres espèces avicoles, s'il est certain qu'elles seront prochainement séquencées, la construction de puces SNP à haut débit commerciales reste peu probable à court terme, faute de marché suffisant, à l'exception peut-être du canard pékin. Pour ces espèces, la chute rapide des coûts de 
séquençage pourrait toutefois conduire à sauter l'étape de mise au point d'une puce commerciale avec la mise en œuvre d'une sélection génomique sur puce SNP « à façon ».

Chez les volailles, contrairement aux bovins laitiers, les gains attendus par réduction de l'intervalle de génération sont faibles, hormis sur les caractères mesurés tardivement comme la ponte. A l'inverse, la précision des valeurs génétiques estimées pourrait être fortement augmentée par l'utilisation des informations génomiques. Dans un contexte d'évolution forte des objectifs de sélection vers plus de durabilité (Jez et al 2010), l'amélioration génétique de « nouveaux » caractères pourrait s'en trouver facilitée (bien-être, résistance aux pathogènes, robustesse). Par ailleurs, l'opportunité est réelle de modifier l'objectif d'un progrès génétique en race pure en un progrès génétique pour une expression en pro- duction (croisement et interaction génotype $\times$ milieu).

Les coûts inhérents à la sélection génomique sont élevés. Le prix d'un génotypage avec la puce poule 600k SNP est actuellement d'environ $200 €$. Si le prix d'obtention d'une population de référence génotypée et phénotypée est difficilement compressible, le prix du génotypage de la population en sélection peut être diminué. A partir de la liste des SNP entrant dans l'équation de prédiction des VGE, une puce dédiée peut être mise au point avec quelques milliers de SNP. Cette technique est, par exemple, appliquée chez les bovins laitiers pour le génotypage en routine des vaches : $7 \mathrm{k}$ SNP pour un coût d'environ $35 €$. Toutefois, la comparaison défavorable souvent avancée entre prix du reproducteur et prix du génotypage doit être nuancée par la diffusion très forte au sein des schémas de sélection pyramidaux.
En effet, tout gain de progrès génétique à l'étage de sélection connaît une diffusion énorme à l'étage de production : par exemple, environ 275000 poules pondeuses sont issues d'un coq de race pure et vont produire 85 millions d'œufs au cours de leur carrière. La multiplication des euros est donc une réalité, mais sa répartition dans le schéma de sélection remet en cause les organisations actuelles.

De nombreuses questions restent donc en suspens et, à l'image du travail réalisé chez le porc par Tribout et al (2011), la modélisation des schémas de sélection avicole reste une étape préalable indispensable pour évaluer les gains attendus dans telle ou telle situation. A fortiori si les évolutions de structures envisagées sont considérables et nécessitent des investissements lourds et une réorganisation complète des schémas de sélection.

\section{Références}

Aguilar I., Misztal I., Johnson D.L., Legarra A., Tsuruta S., Lawlor T.J., 2010. Hot topic: A unified approach to utilize phenotypic, full pedigree, and genomic information for genetic evaluation of Holstein final score. J. Dairy Sci., 93, 743-752.

Colombani C., Croiseau P., Fritz S., Guillaume F., Legarra A., Ducrocq V., Robert-Granié C., 2012. A comparison of partial least squares (PLS) and sparse PLS regressions in genomic selection in French dairy cattle. J. Dairy Sci., 95, 2120-2131.

Coudurier B., 2011. Contraintes et opportunités d'organisation de la sélection dans les filières porcine et avicole. In : Numéro spécial, Amélioration génétique. Mulsant P., Bodin L., Coudurier B., Deretz S., Le Roy P., Quillet E., Perez J.M. (Eds). INRA Prod. Anim., 24, 307-322.

Daetwyler H.D., Villanueva B., Bijma P., Wooliams J.A., 2007. Inbreeding in genomewide selection. J. Anim. Breed. Genet., 124, 369-376.

Dekkers J.C.M., Chakraborty R., 2004. Optimizing purebred selection for crossbred performance using QTL with different degrees of dominance. Genet. Sel. Evol., 36, 297-324.

Dekkers J.C.M., 2007. Marker-assisted selection for commercial crossbred performance. J. Anim. Sci., 85, 2104-2114.

De Roos A.P.W., Hayes B.J., Goddard M.E., 2009. Reliability of genomic predictions across multiple populations. Genetics, 183, 1545-1553.

Fernando R.L., Grossmann M., 1989. Marker assisted selection using best linear unbiased prediction. Genet. Sel. Evol., 21, 467-477.

Fisher R.A., 1918. The Correlation between Relatives on the Supposition of Mendelian Inheritance. Philosophical Transactions of the Royal Society of Edinburgh, 52, 399-433.
Fritz S., Guillaume F., Croiseau P., Baur A., Hoze C., Dassonneville R., Boscher M.Y., Journaux L., Boichard D., Ducrocq V., 2010. Mise en place de la sélection génomique dans les trois principales races françaises de bovins laitiers. Renc. Rech. Rum., 17, 455-458.

Goddard M.E., 1992. A mixed model for analyses of data on multiple genetic markers. Theor. Appl. Genet., 83, 878-886.

Goddard M.E., 2009. Genomic selection: prediction of accuracy and maximisation of long term response. Genetica, 136, 245-257.

Guillaume F., Fritz S., Croiseau P., Legarra A., Robert-Granié C., Colombani C., Patry C., Boichard D., Ducrocq V., 2009. Modèles d'évaluation génomique : application aux populations bovines laitières françaises. Renc. Rech. Rum., 16, 399-406.

Guillaume F., Boichard D., Ducrocq V., Fritz S., 2011. Utilisation de la sélection génomique chez les bovins laitiers. In : Numéro spécial, Amélioration génétique. Mulsant P., Bodin L., Coudurier B., Deretz S., Le Roy P., Quillet E., Perez J.M. (Eds). INRA Prod. Anim., 24, 363-368.

Habier D., Fernando R.L., Dekkers J.C.M., 2007. The Impact of Genetic Relationship Information on Genome-Assisted Breeding Values. Genetics, 177, 2389-2397.

Habier D., Fernando R.L., Kizilkaya K., Garrick D.J., 2011. Extension of the bayesian alphabet for genomic selection. BMC Bioinformatics, 12,186

Haley C.S., Visscher P.M., 1998. Strategies to Utilize Marker-Quantitative Trait Loci Associations. J. Dairy Sci., 81, Suppl. 2, 85-97.

Hayes B.J., Bowman P.J., Chamberlain A.J., Goddard M.E., 2009. Genomic selection in dairy cattle: Progress and challenges. J. Dairy Sci., 92, 433-443.
Ibanez-Escriche N., Fernando R.L., Toosi A., Dekkers J.C.M., 2009. Genomic selection of purebreds for crossbred performance. Genet. Sel. Evol., 41, 12-21.

Jez C., Beaumont C., Magdelaine P., 2010. La filière avicole française à l'horizon 2025. Poultry Sci., 67, 105-114.

Kinghorn B.P., Hickey J.M., van der Werf J.H.J., 2010. Reciprocal Recurrent Genomic Selection for Total Genetic Merit in Crossbred Individuals. In: Proc. $9^{\text {th }}$ WCGALP, Leipzig, Germany. Comm. 36.

Lande R., Thompson R., 1990. Efficiency of marker-assisted selection in the improvement of quantitative traits. Genetics, 124, 743-776.

Legarra A., Robert-Granié C., Manfredi E., Elsen J.M., 2008. Performance of Genomic Selection in Mice. Genetics, 180, 611-618.

Long N., Gianola D., Rosa G.J.M., Weigel K.A., Avendaño S., 2007. Machine learning classification procedure for selecting SNPs in genomic selection: application to early mortality in broilers. J. Anim. Breed. Genet., 124, 377-389.

Lund M.S., De Roos A.P.W., De Vries A.G., Druet T., Ducrocq V., Fritz S., Guillaume F., Guldbrandtsen B., Liu Z., Reents R., Schrooten C., Seefried M., Su G., 2010. Improving genomic prediction by EuroGenomics collaboration. In: Proc. $9^{\text {th }}$ WCGALP, Leipzig, Germany. Comm. 880.

Meuwissen T.H.E., Hayes B.J., Goddard M.E., 2001. Prediction of Total Genetic Value Using Genome-Wide Dense Marker Maps. Genetics, 157, 1819-1829.

Robert-Granié C., Legarra A., Ducrocq V., 2011. Principes de base de la sélection génomique. In : Numéro spécial, Amélioration génétique. Mulsant P., Bodin L., Coudurier B., Deretz S., Le Roy P., Quillet E., Perez J.M. (Eds). INRA Prod. Anim., 24, 331-340. 
Ruane J., Colleau J.J., 1995. Marker assisted selection for genetic improvement of animal populations when a single QTL is marked. Genet. Res., 66, 71-83.

Solberg T.R., Sonesson A.K., Wooliams J.A., Meuwissen T.H.E., 2008. Genomic selection using different marker types and densities. J. Anim. Sci., 86, 2447-2454.

Solberg T.R., Sonesson A.K., Wolliams J.A., Odegard J., Meuwissen T.H.E., 2009. Persistence of accuracy of genome-wide breeding values over generations when including a polygenic effect. Genet. Sel. Evol., 41, 53 .

Soller M., 1994. Marker assisted selection - an overview. Anim. Biotechnol., 5, 247-252.
Sonesson A.K., Meuwissen T.H.E., 2009 Testing strategies for genomic selection in aquaculture breeding programs. Genet. Sel. Evol., 41, 37.

Tribout T., Bidanel J.P., Phocas F., Schwob S. Guillaume F., Larzul C., 2011. La sélection génomique : principe et perspectives d'utilisation pour l'amélioration des populations porcines. Journ. Rech. Porcines, 43, 13-26.

VanRaden P.M., 2008. Efficient Methods to Compute Genomic Predictions. J. Dairy Sci., 91, 4414-4423

Wolc A., Arango J., Settar P., Fulton J.E. O'Sullivan N.P., Preisinger R., Habier D., Fernando R., Garrick D.J., Dekkers J.C.M., 2011a. Persistence of accuracy of genomic estimated breeding values over generations in layer chickens. Genet. Sel. Evol., 43, 23

Wolc A., Stricker C., Arango J., Settar P., Fulton J.E., O'Sullivan N.P., Preisinger R., Habier D., Fernando R., Garrick D.J., Lamont S.J., Dekkers J.C.M., 2011b. Breeding value prediction for production traits in layer chickens using pedigree or genomic relationships in a reduced animal model. Genet. Sel. Evol., 43, 5.

Vignal A., 2011. Etat actuel du séquençage et de la connaissance du génome des espèces animales. In : Numéro spécial, Amélioration génétique. Mulsant P., Bodin L., Coudurier B., Deretz S., Le Roy P., Quillet E., Perez J.M. (Eds). INRA Prod. Anim., 24, 387-404.

\section{Résumé}

La sélection génomique repose sur l'évaluation des valeurs génétiques des candidats à la sélection par un " score moléculaire " calculé d'après leurs génotypes en un très grand nombre de marqueurs de l'ADN. Dans une première étape, les effets des marqueurs sont estimés sur une population de référence génotypée et phénotypée pour les caractères à améliorer. La table des valeurs génotypiques ainsi établie est utilisée pour calculer les valeurs génétiques des candidats au cours des générations suivantes, qui peuvent alors n'être que génotypées. En quelques années, cette stratégie a été mise en place chez les bovins laitiers. Les opérateurs de la sélection dans les autres filières animales ont assisté à cette révolution et se posent légitimement la question de l'intérêt pour eux d'entrer dans cette démarche. La disponibilité d'une puce «poule» de 600k SNP permet d'ores et déjà d'appliquer une sélection génomique dans les filières poule pondeuse et poulet de chair. Pour les autres espèces avicoles, les outils de génotypage à haut débit sont en cours de développement. Quelle que soit l'espèce, trois composantes du progrès génétique peuvent être améliorées. L'intensité de sélection peut être augmentée pour les caractères non mesurables en routine (qualités des produits, efficacité alimentaire, résistance aux maladies). La précision des valeurs génétiques peut être augmentée, notamment pour les mâles dans le cas des caractères exprimés uniquement par les femelles. Enfin, l'intervalle de génération peut être diminué grâce à une évaluation précoce des candidats, notamment en sélection des poules pondeuses. Par ailleurs, l'évaluation génomique offre pour la première fois la possibilité de sélectionner des individus de race pure de l'étage de sélection pour leur valeur en croisement et dans le milieu de production. Les coûts de mise en ouvre de la sélection génomique sont élevés, en raison de la taille de la population de référence nécessaire à une évaluation précise, du nombre élevé de candidats à génotyper et de la variété des populations à sélectionner. Toutefois, chez les volailles, l'énorme diffusion des reproducteurs pourrait permettre de compenser ces coûts.

\section{Abstract}

Genomic selection: What are the perspectives for poultry industries?

Genomic selection is based on an evaluation of the genetic values of the candidates for selection through a «molecular score» calculated from their genotypes for a great number of DNA markers. A first step consists in estimating the effects of the markers on a reference population that has been genotyped and phenotyped for the traits that need improving. The establishment of this table of genotypic values allows for the calculation of the genetic values of the candidates in the subsequent generations, for which genotyping might then prove sufficient. This strategy was implemented in dairy cattle in just a few years. The operators in charge of selection in the other animal production sectors have witnessed this change and hence legitimately regard this approach as useful input for them. The existence of a 600k SNP chip for the chicken now allows for the application of genomic selection in layers and broilers. Tools for high-throughput genotyping in other poultry species are being developed. There are three components of genetic progress that can be improved, regardless of the species. First, selection intensity can be increased for traits non-measurable in routine (product quality, feed efficiency, resistance to diseases). Then, the accuracy of genetic values can be improved, especially for males whenever traits are expressed by females only. Finally, the generation interval can be reduced through early evaluation of candidates (egg production). What is more, genomic evaluation provides, for the first time, the opportunity to select pure-bred individuals from the selection nucleus for their usefulness in crossbreeding and production. The implementation costs of genomic selection are quite high. This is due to the size of the reference population needed for accurate evaluation, the huge number of genotyping operations to be carried out on candidates and the great diversity of populations to be selected. However, use of large numbers of breeding animals in the poultry industry could help counterbalance these costs.

LE ROY P., CHAPUIS H., GUÉMENÉ D., 2014. Sélection génomique : quelles perspectives pour les filières avicoles ? INRA Prod. Anim., 27, 331-336. 